\title{
Optical Imaging Method for Determining Symptoms Severity of Cassava Mosaic Disease
}

\author{
Benjamin Anderson ${ }^{1}$, Moses Jojo Eghan ${ }^{1}$, Elvis Asare-Bediako² \& Paul Kingsley Buah-Bassuah ${ }^{1}$ \\ ${ }^{1}$ Laser and Fibre Optics Centre, Department of Physics, University of Cape Coast, Ghana \\ ${ }^{2}$ Department of Crop Science, School of Agricultural Science, University of Cape Coast, Ghana \\ Correspondence: Benjamin Anderson, Laser and Fibre Optics Centre, Department of Physics, University of Cape \\ Coast, Ghana. E-mail: banderson@ucc.edu.gh
}

Received: September 8, 2015 Accepted: September 24, 2015 Online Published: October 10, 2015

doi:10.5539/apr.v7n6p34

URL: http://dx.doi.org/10.5539/apr.v7n6p34

\begin{abstract}
Cassava mosaic disease (CMD) is a major constraint to cassava production in cassava growing regions. Severity of CMD symptoms on cassava leaves is usually assessed visually using an arbitrary scale, which is semi-qualitative, and does not represent the actual surface area of diseased leaf. The objective of this study was to develop a quantitative method of assessing the severity of CMD. A combination of polarimeteric digital colour images, $\mathrm{L}^{*} \mathrm{a} * \mathrm{~b} *$ colour model and $K$-means clustering algorithm were used to determine the areas of CMD symptoms and healthy areas on leaves. The severity of CMD on a leaf is determined by computing the percentage of the CMD symptomatic area to the total leaf area. The analysis provides relatively fast and accurate classification of Cassava mosaic diseased leaves. The proposed method will enable plant scientists to obtain accurate and reliable data, forming the basis for better decision making.
\end{abstract}

Keywords: Cassava mosaic disease, Digital camera, Polarimetric image, L*a*b* colour model, k-means clustering, symptoms severity

\section{Introduction}

Cassava mosaic disease (CMD) has received much attention in recent years due to its destructive nature to the economic yield of cassava as cassava provides the world's third calories (Nassar \& Ortiz, 2010; Alves, 2002; FAOSTAT, 2009). This disease is caused by a virus in the genus Begomovirus, (family Geminiviridae), and is the most common viral disease of cassava, especially in Africa (Fauquet \& Stanley, 2003; Sseruwagi, Sserubombwe, Legg, Ndunguru, \& Thresh, 2004; Thresh \& Cooter, 2005). It destroys the chlorophyll pigments plants need for photosynthesis, and therefore limits the plants growth, resulting in poor yields. Drastic reduction in the yield of cassava (Fargette, Fauquet, \& Thouvenel, 1988; Geddes, 1990; Thresh, Otim-Nape, Legg, \& Fargette, 1997; Calvert \& Thresh, 2002; Sseruwagi, Sserubombwe, Legg, Ndunguru, \& Thresh, 2004), is largely attributed to the severity of disease symptoms (Osiru, Sserbombwe, Sseruwagi, Thresh, \& Otom-Nape, 1999; Anderson et al., 2012). These symptoms range from uniform green to yellow mosaic patterns coupled with leaf distortions, reductions, and stunting as secondary effects (Alabi, Kumar, \& Naidu, 2011; Thresh \& Cooter, 2005). Appearance of a virus induced chlorosis and malformation of leaflets, which is asymmetric about the midrib distinguishes CMD symptoms from those of mineral deficiency or cassava green mite damage. The severity of the disease, which indicates the extent of symptom development (or the degree of symptom expression), is one of the parameters that are monitored in the epidemiological studies of CMD.

Severity of plant disease is the area of the plant tissue that is diseased relative to the total area of the tissue. However, there are several different methods of measuring the severity of plant disease: visual rating, imaging analysis, and hyperspectral imagery (Bock, Poole, Parker, \& Gottwald, 2010). In visual rating, the assessment process is quick and requires no equipment and the use of assessment aids can improve the results remarkably. Nonetheless, raters are prone to various illusions bringing about decreased accuracy and their services tend to be expensive. Visual rating, by raters, is the standard method currently used for determining CMD severity, with an arbitrary scale of either 0 - 4 (Fauquet \& Fargette, 1990) or 1- 5 (Cours, 1951; Hahn, Terry, \& Leuschner, 1980; Fauquet \& Fargette, 1990; International Institute for Tropical Agriculture, 1990; Sseruwagi, Sserubombwe, Legg, Ndunguru, \& Thresh, 2004; Ariyo, Dixon, \& Atiri, 2005). However, this approach is semi-qualitative and somewhat subjective and does not measure the disease severity accurately. Furthermore, the scale-based estimates are limited by personal, 
physical, and environmental factors. The disease may occur in areas that are not visible to be observed, and different scales may result in different severity, which may be confusing. Grid counting method had been proposed to improve the accuracy, however, it is found to be clumsy and time consuming procedure.

Image analysis offers a lot of important visual information from the colours (MATLAB, 2010). Performing this analysis for plant disease assessment is a very quick procedure and requires relatively inexpensive equipment. Existing technology makes the assessment both reliable and accurate and is extremely powerful when a good automated system is developed. For instance, using image segmentation method in the analysis will provide important information for visualisation and quantification (Haralick \& Shapiro, 1985; Gonzalez \& Woods, 2010), which can be applied to any multispectral image (Gonzalez \& Woods, 2010). Digital colour image segmentation is adaptable to uncalibrated colour cameras and device-independent colour models, and is computationally inexpensive (Gonzalez \& Woods, 2010). These features have made digital colour image segmentation an attractive technique. The technique has been used extensively in many research and applications (Deng \& Manjunath, 2001; Freixenet, Munoz, Raba, Marti, \& Cu, 2002; Wang, Yang, \& Tian, 2008). Aduwo, Mwebaze and Quin (2010) report of image analysis on CMD, using automated vision diagnosis, which is based on the colour and shape of the leaf. For hyperspectral imagery, massive amounts of data and information about the target are acquired simultaneously (Bock, Poole, Parker, \& Gottwald, 2010). However, the enormous data size can slow the capturing process. Also, the technology is still new and requires substantial training and expertise for its use, making it an expensive venture.

In this paper, a new digital colour image analysis method for determining the severity of CMD of a leaf is presented. This method is based on analysing an image taken through polarization filters using $\mathrm{L}^{*} \mathrm{a} * \mathrm{~b} *$ colour segmentation model and $K$-means clustering. The polarization filters are set at an orientation that best improves image colour contrast. This method does not measure the virus concentration or the type of virus.

\section{Methodology}

\subsection{Samples}

Cassava mosaic disease infected and healthy leaves were collected from 5-month-old cassava plants grown at the School of Agriculture Teaching and Research Farm, University of Cape Coast, Ghana. The infected leaves were randomly selected throughout the field from different varieties without systematic inoculation treatment.

\subsection{Polarimeteric Digital Colour Imaging}

Setup for polarimeteric digital colour imaging of the cassava leaves is shown in Figure 1. The camera is HP Photosmart M415 (FCLSD-0504, Hewlett-Packard Development Company, L.P). It has a sensor resolution of 2620 X 1984 effective pixels count and provides images with 12 bits per colour channel, and a macro oval builtin flash that offers shadowless and uniform illumination. The images are displayed on a $38.1 \mathrm{~mm}$ built-in colour monitor. The camera is connected to a Laptop computer.

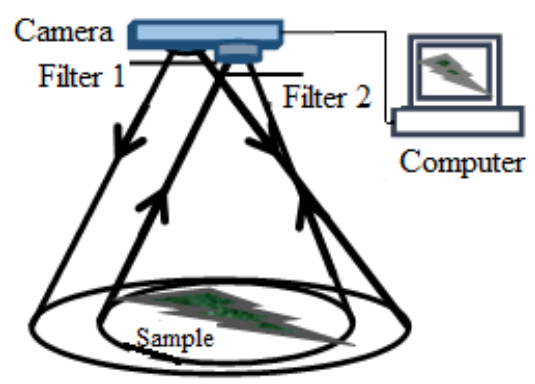

Figure 1. A setup for polarimeteric digital colour imaging of the cassava leaves. Filters 1 serves as a polarizer in front of the built-in flash to polarize the incident light. Filter 2 serves as an analyzer in front of the detector lens and placed orthogonal to filter 1 in order to cutoff the specular reflected light. It allows only the diffused reflected light to be transmitted

Filters 1 and 2 are linear polarizer and analyzer (Model NT45-667, Edmund Industrial Optics, Barrington, NJ) orthogonally arranged and placed in front of the flash and the camera lens respectively to reduce the glare caused by specular reflectance from the surfaces of the leaves. The leaves were placed on a black cardboard to reduce back reflections. 
Polarimeteric digital colour images were acquired from the same distance of $500 \mathrm{~mm}$ in a uniform environment and stored in tagged image file format (TIFF). To ensure that the images taken contain only the leaf and the background, the full zoom of the camera was used. For reproducibility, the camera, the polarizer and the analyzer were mounted on a retort stand. In all, 64 infected and 10 healthy leaves were imaged.

\subsection{Image Segmentation}

The original polarimeteric digital colour image, which is in RGB colour space and device dependent, was converted into Commission International de l'Eclairage (CIE) recommended L*a*b colour model (Gonzalez \& Woods, 2010). In the conversion process, the pixel values in the RGB colour space were first transformed into device independent CIE XYZ tristimulus values using a conversion matrix (Eqn. 1) as if they were acquired under average daylight illumination.

$$
\left[\begin{array}{l}
X \\
Y \\
Z
\end{array}\right]=\left[\begin{array}{lll}
0.412453 & 0.357580 & 0.180423 \\
0.212627 & 0.715160 & 0.072169 \\
0.019334 & 0.119193 & 0.950227
\end{array}\right]\left[\begin{array}{l}
R \\
G \\
B
\end{array}\right]
$$

The CIE XYZ tristimulus values were then converted into $\mathrm{L}^{*}, \mathrm{a}^{*}$ and $\mathrm{b}^{*}$ chromaticity layers based on a white standard that gives $X_{n}, Y_{n}$, and $Z_{n}$ values described by:

$$
\begin{gathered}
L^{*}=116\left[\frac{Y}{Y_{n}}\right]^{1 / 3}-16 \\
a^{*}=500\left(\left[\frac{X}{X_{n}}\right]^{1 / 3}-\left[\frac{Y}{Y_{n}}\right]^{1 / 3}\right) \\
b^{*}=200\left(\left[\frac{Y}{Y_{n}}\right]^{1 / 3}-\left[\frac{Z}{Z_{n}}\right]^{1 / 3}\right)
\end{gathered}
$$

Using only $\mathrm{a}^{*}$ and $\mathrm{b}^{*}$ colour components, the leaves were segmented using the $K$-means clustering algorithm (MATLAB, 2010).

\subsection{The K-means Clustering Algorithm}

The $K$-means clustering algorithm is one of the most standardized and resourceful clustering methods for unsupervised learning tasks (Lloyd, 1957, MacQueen, 1967; Hartigan \& Wang, 1979; Jain \& Dubes, 1988; MATLAB, 2010). The $K$-means clustering starts with an initial partition with $K$ centroids of clusters, and the assignment of patterns to characterise the data. The number of clusters is determined by the number of colour patterns in the image. The clusters are obtained by minimising the sum of squared errors of the pixels in the data matrix with fixed number, $\mathrm{K}$, of clusters as given in equation 3 ,

$$
J(C)=\sum_{k=1}^{K} \sum_{p_{i} \in c_{k}}\left\|p_{i}-\mu_{k}\right\|^{2}
$$

where $C=\left\{c_{k}, k=1, \ldots, K\right\}$ is the set of $K$ clusters, $\left(p_{1}, \ldots, p_{n}\right)=P$ is the data matrix and $\mu_{k}=\sum_{p_{i} \epsilon c_{k}} p_{i} / n_{k}$ is the centroids of the cluster $c_{k}$ and $n_{k}$ is the number of points in $c_{k}$.

The algorithm first assigns each pixel to a cluster that has the closest centroid. When all the pixels had been assigned, the positions of the $K$ centroids are recalculated, and the process is repeated until the centroids no longer move. Using the result from the $K$-means clustering each pixel in the images is labelled. $K$ colour images are then created by segmenting the image by colour using the pixel labels.

\subsection{Disease Severity Determination}

Using the $K$-means segmented image, the severity of a CMD leaf, DS, is determined as a ratio of the CMD symptomatic area, SA, to the total leaf area, TA, which is expressed as a percentage in equation 4 ,

$$
D S=\left(\frac{S A}{T A}\right) 100=\left(\frac{\sum P D}{\sum P T}\right) 100
$$

where $\sum P D$ and $\sum P T$ represent the sum of pixels in SA, and sum of the pixels in TA respectively. DS was obtained by counting the pixels in SA and TA. The percentage error involved in each calculation is then determined. The whole image analysis algorithm to determine the percentage severity of cassava mosaic disease from a cassava leaf is summarized as a flowchart in Figure 2. 


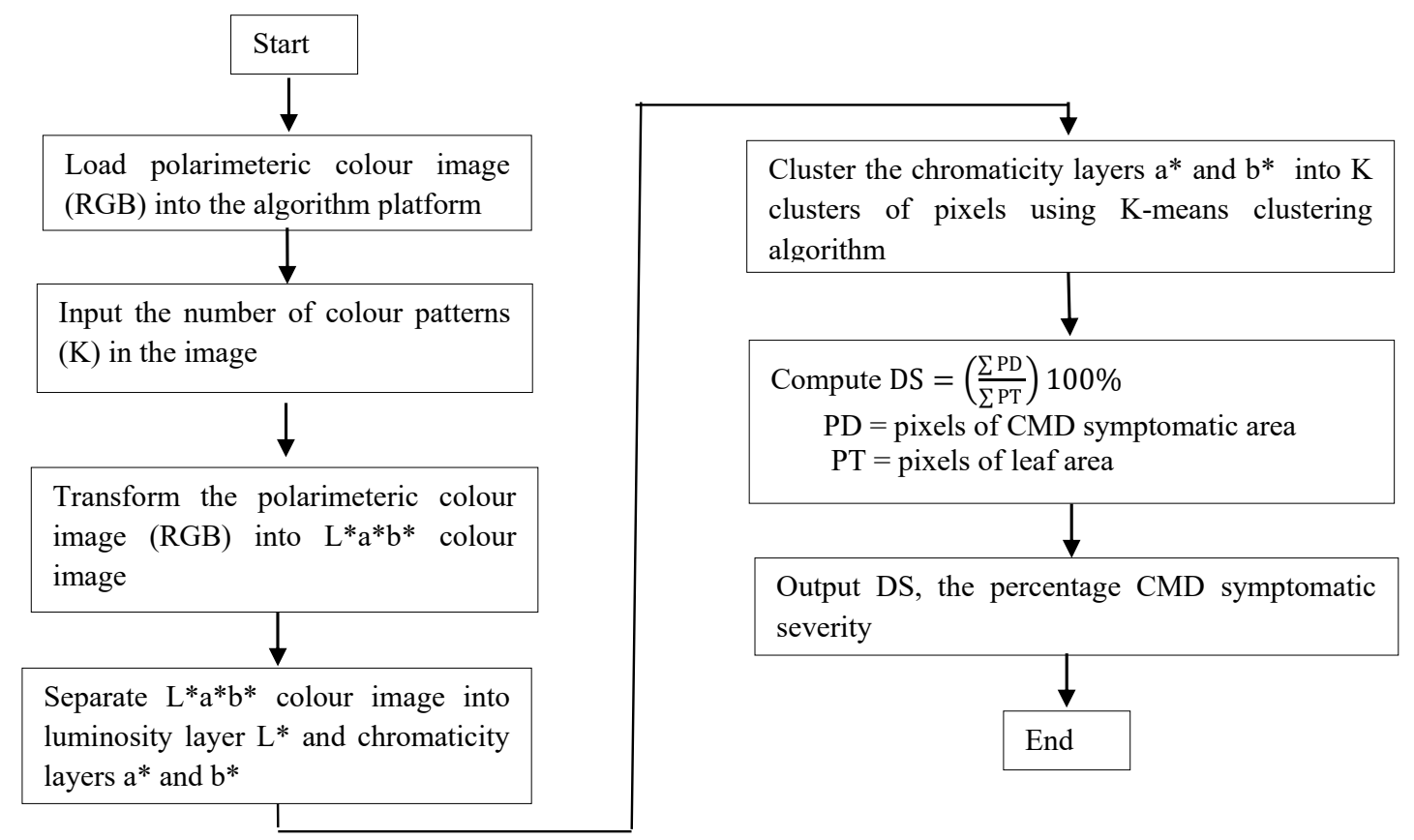

Figure 2. Image analysis of cassava leaves to determine the percentage severity of cassava mosaic disease algorithm expressed as a flowchart

\section{Results and Discussions}

Figure 3a shows some of the polarimeteric digital colours images of (i) healthy leaf and (ii - v) CMD infected leaves recorded in RGB colour space. The images contain information on the physiological state of the leaf pigmentation in the form of diffuse reflectance. The specular reflected lights, which were removed with the orthogonally arranged polarization filters 1 and 2, contain only surface morphological information about the leaf (Wolff, 1989), and tend to impair the observation of the leaf colour information provided by the diffused reflectance from the subsurface structures. Figure $3 b$ shows the corresponding images in CIE $L^{*} a * b *$ colour space when the CIE $\mathrm{L}^{*} \mathrm{a} * \mathrm{~b} *$ colour model was applied. The motivation is that, while the RGB images are machine dependent, that is, depends on the type of digital camera used in imaging, the CIE L*a*b* colour space is machine independent. In addition, the CIE $\mathrm{L}^{*} \mathrm{a} * \mathrm{~b} *$ colour space is perceptually uniform, enabling even images taken under varying experimental conditions to be compared.

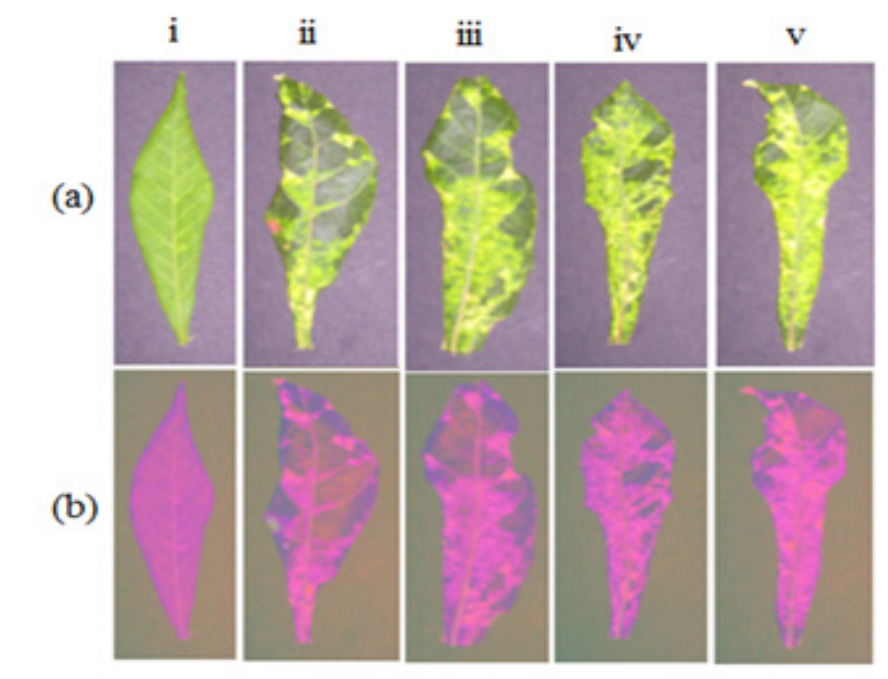

Figure 3. (a) Unprocessed polarimeteric digital colours images of (i) healthy leaf and (ii-v) cassava mosaic infected leaves in red green and blue (RGB) colour space and (b) the corresponding images in CIE $\mathrm{L}^{*} \mathrm{a}^{*} \mathrm{~b}^{*}$ colour space when $\mathrm{L}^{*} \mathrm{a} * \mathrm{~b} *$ colour model is applied 
The CIE L*a*b* colour images have a lower image contrast compared with that of the RGB images, because of the reduced brightness resulting from the relative removal of the specular reflections. However, the colour contents, which is the most important information describing the physiological state of the leaves, are present.

Figure 4 shows the various layers; luminosity, $\mathrm{L}^{*}$ or brightness layer, and chromaticity layers $\mathrm{a}^{*}$ and $\mathrm{b}^{*}$, in the CIE L*a*b* colour space from the leaves. These chromaticity layers $a^{*}$ and $b^{*}$ contain the entire colour (or spectral) information in the leaves.

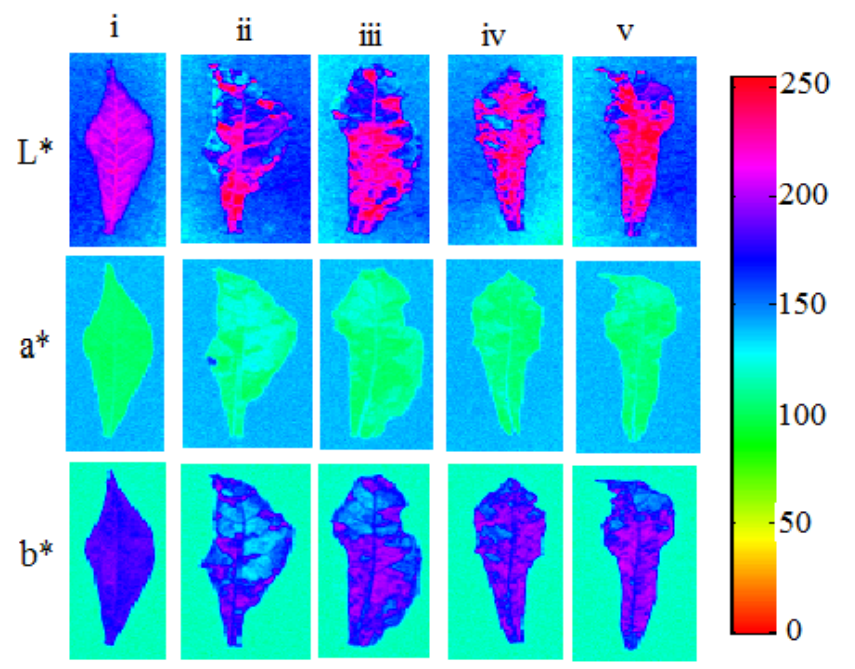

Figure 4. Separate layers, luminosity, $\mathrm{L}^{*}$ and chromaticity layers $\mathrm{a}^{*}$ and $\mathrm{b}^{*}$, in the CIE $\mathrm{L}^{*} \mathrm{a} \mathrm{b}^{*}$ colour space for (i) healthy leaf and (ii-v) CMD-infected leaves. The $\mathrm{a}^{*}$ layer specifies where a colour falls along the red-green axis, and $b^{*}$ layer specifying where the colour falls along blue-yellow axis

The chromaticity layer $\mathrm{a}^{*}$ specifies where the colour of the leaf falls along the red-green axis, while the $\mathrm{b}^{*}$ layer specifies the colour of the leaf along blue-yellow axis. The dominant red colours in the $\mathrm{L}^{*}$ layer represent regions in the leaves with the highest diffused reflections. Unlike the healthy leaf, the bright region in the CMD infected leaves start from a small area and spread outwards. These red colours describe the regions in the leaves where the scattered light produced the highest intensity.

The $\mathrm{a}^{*}$ layer of the leaves has lower chromaticity values compared with that of the $\mathrm{b}^{*}$ layer. This indicates that the leaves reflected little diffused light within red-green region compared with the reflections from the blue-yellow region. The pink regions in the $\mathrm{b}^{*}$ layer describe the diffuse reflections from the yellow portions of the original image, confirming the fact that yellow reflects more light to the human eye than any other colour in the visible region (Hahn, Terry, \& Leuschner, 1980).

Only the chromaticity components, $\mathrm{a}^{*} \mathrm{~b}^{*}$, is used in order to eliminate any leftover glare, shadow and other factors that may affect the images during the imaging process. It also brings more appropriate visual colour characteristics of human beings that may be hidden from the naked human eye, which is the main determining factor of the plant researchers. All this is because the human vision is more sensitive to colour (Gonzalez \& Woods, 2010). The colour criterion was used to construct these realistic classifiers because the spectral information is the most significant in the image data and is adequately representing the physical phenomena (Haralick \& Shapiro, 1985; Gonzalez \& Woods, 2010).

Three main colours, black, green, and yellow are observed in the RGB colour image, define the $K$ in the $K$-means clustering algorithm. This is because they are the main colours found in the polarimeteric digital colour images to determine the natural spectral grouping present in the image. The $K=3$-means clustering treats the pixels, with ' $\mathrm{a}^{*}$ ' and ' $\mathrm{b}^{*}$ ' values, as objects, and cluster the patterns such that the square errors of the objects within each cluster are minimised. For every object, the clustering returned an index corresponding to a cluster. The pixels in the image were then labelled with its cluster index by assigning to the cluster whose arbitrary mean vector is the closest. The procedure continues until there was no significant change in the location of the class centroid vectors between 
the successive iterations of the algorithms. The chromaticity colour layers using the pixel labels then separated the objects in the image.

Figure 5 shows the comparison of some of the polarimetric digital colour images of (i) healthy and (ii-v) the green and yellow portions of cassava mosaic diseased leaves after using the chromaticity layers $\mathrm{a}^{*}$ and $\mathrm{b}^{*}$ and the kmeans clustering. Apart from the yellow curve in (b)(i), which shows the boundary between the background and the leaf, the yellow and green portions of the images in (b) and (c) conform well with their original images in (i).This is taken care of with normalization when the percentage severity is evaluated.

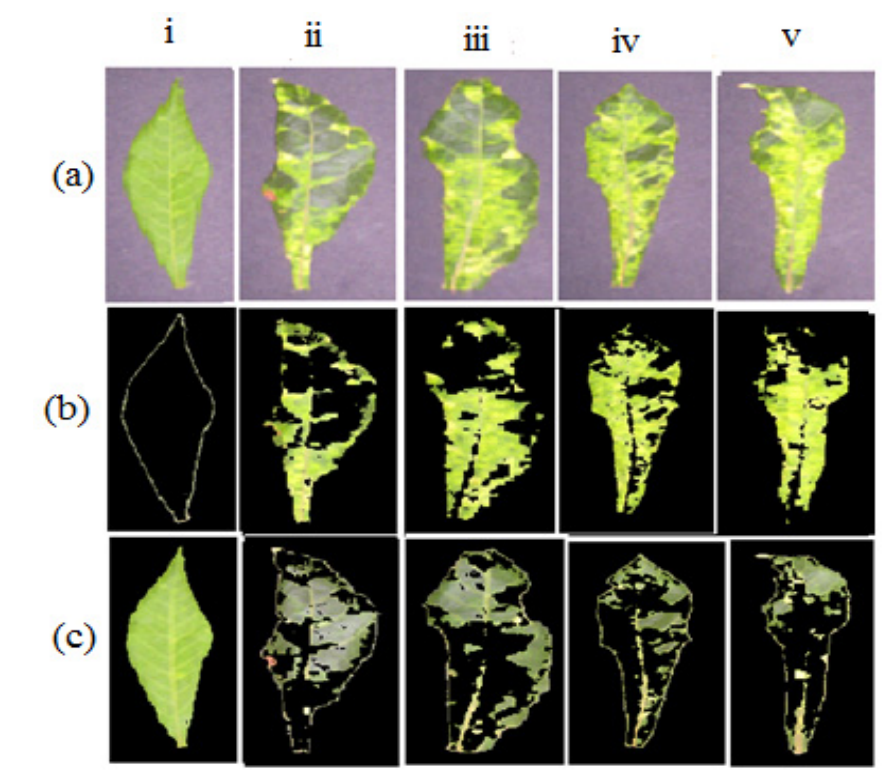

Figure 5. Comparison of polarimeteric digital colour images of (i) healthy leaf and (ii-v) CMD infected leaves with the corresponding analysed images. (a) shows the unprocessed polarimeteric digital colour images and (b) the extracted yellow patches, and (c) green patches from the leaves

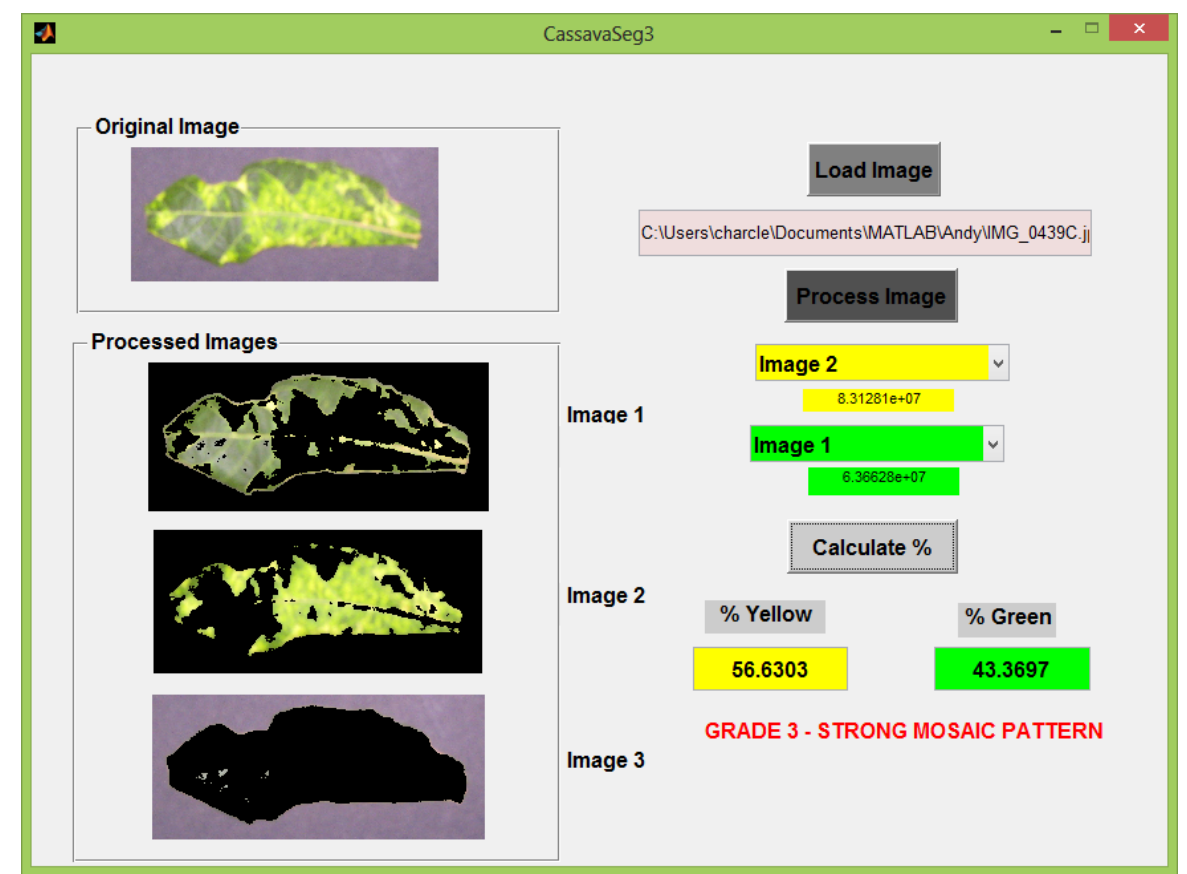

Figure 6. Illustration of Graphical User Interface (GUI) developed using MATLAB codes to analyse any polarimeteric digital colour image of a cassava leaf and estimate the percentage CMD severity. $\mathrm{K}=3$ - means clustering is illustrated under the processed images and the knobs needed to load and process the image is found on the right-hand side of the GUI 
The significance of this segmentation technique is that the separated regions do not intersect each other in any way and each region meets the reliability conditions in specific regions (Gonzalez \& Woods, 2010; Patil \& Bodhe, 2010). We show in Figure 6 an illustration of a Graphical User Interface (GUI) developed, using MATLAB codes, to automate the whole analysis process. Processing time for using this GUI on a digital leaf image of $300 \mathrm{x} 200 \mathrm{x}$ 3 pixel counts was 10 seconds on average.

Table 1 shows some polarimeteric digital colour images of the cassava mosaic disease infected leaves and their corresponding percentage symptoms severity obtained from the analysis. On the average, percentage error for calculating the percentage symptoms severity of cassava leaves for all the samples considered are in the orders of $10^{-5}$.

Table 1. Some polarimeteric digital colour images of the Cassava mosaic disease infected leaves and their corresponding percentage symptoms severity

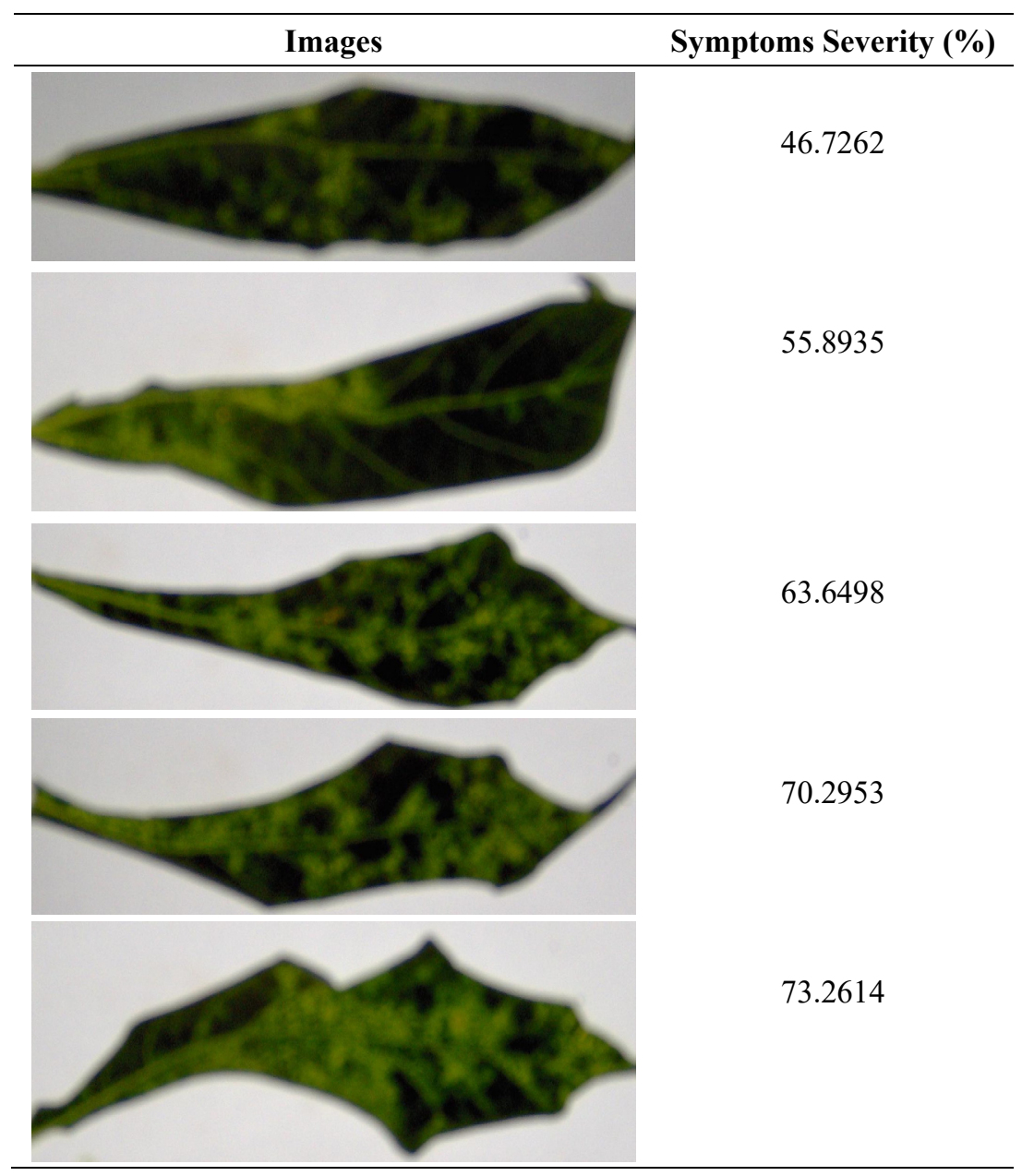

\section{Conclusions}

The task of determining the severity of cassava mosaic disease (CMD) affecting cassava leaves using image analysis has been demonstrated. The method is based on polarimeteric digital colour image, $\mathrm{L}^{*} \mathrm{a} * \mathrm{~b} *$ colour space and K-means clustering algorithms. In this approach, a commercial digital camera was used to acquire the image. The method quantifies the diseased area as a percentage of the total leaf area in order to make objective conclusions about the severity of CMD. This method is new, simple, and objective for classifying cassava leaves in terms CMD severity and does not need a specialist, because it is easy to use and do not require any special training. This method will enable plant pathologists to make better-informed decisions as the data is more accurate and precise.

\section{Acknowledgements}

The authors are grateful to the Associate Scheme and the Office of External Activities (OEA) of Abdus Salam ICTP Trieste, Italy for their financial support during their stay at the centre and the use of the library and computing 
facilities to write this paper. We are also grateful to International Programme for Physical Sciences (IPPS), International Sciences Programme (ISP), Uppsala University, Sweden for the financial support.

\section{References}

Aduwo, J. R., Mwebaze, E., \& Quinn, J. A. (2010). Automated Vision-Based Diagnosis of Cassava Mosaic Disease. In Industrial Conference on Data Mining-Workshops (pp. 114-122).

Alabi, O. J., Kumar, P. L., \& Naidu, R. A. (2011). Cassava mosaic disease: A curse to food security in Sub-Saharan Africa. Online. APSnet Features. http://dx.doi.org/10.1094/APSnetFeature-2011-0701

Alves, A. A. C. (2002). Cassava botany and physiology. Cassava: biology, production and utilization, 67-89. http://dx.doi.org/10.1079/9780851995243.0067

Anderson, B., Eghan, M. J., Asare-Bediako, E., \& Buah-Bassuah, P. K. (2012). Violet diode laser-induced chlorophyll fluorescence: a tool for assessing mosaic disease severity in cassava (Manihot esculenta Crantz) cultivars. Environmental technology, 33(3), 367-372. http://dx.doi.org/10.1080/09593330.2011.575184

Ariyo, O. A., Dixon, A. G. O., \& Atiri, G. I. (2005). Whitefly Bemisia tabaci (Homoptera: Aleyrodidae) infestation on cassava genotypes grown at different ecozones in Nigeria. Journal of economic entomology, 98(2), 611617. http://dx.doi.org/10.1093/jee/98.2.611

Bock, C. H., Poole, G. H., Parker, P. E., \& Gottwald, T. R. (2010). Plant disease severity estimated visually, by digital photography and image analysis, and by hyperspectral imaging. Critical Reviews in Plant Sciences, 29(2), 59-107. http://dx.doi.org/10.1080/07352681003617285

Calvert, L. A., \& Thresh, J. M. (2002). The viruses and virus diseases of cassava. In: Hillocks, R. J., Thresh, J. M., \& Bellotti, A. C. (Eds). Cassava: Biology, Production and Utilization (pp. 236-260). Wallingford, UK: CABI Publishing. http://dx.doi.org/10.1079/9780851995243.0237

Cours G. (1951). Le Manioc i Madagascar. Mémoires de l'Institut Scientifique de Madagascar. Série B, Biologie Végétale, 3, 203-400.

Deng, Y., \& Manjunath, B. S. (2001). Unsupervised segmentation of color-texture regions in images and video. IEEE Transactions on Pattern Analysis and Machine Intelligence, 23(8), 800-810. http://dx.doi.org/10.1109/34.946985

Fargette, D., Fauquet, C., \& Thouvenel, J. C. (1988). Yield losses induced by African cassava mosaic virus in relation to the mode and the date of infection. International Journal of Pest Management, 34(1), 89-91. http://dx.doi.org/10.1080/09670878809371216

Fauquet, C. M., \& Stanley, J. (2003). Geminivirus classification and nomenclature: progress and problems. Annals of applied biology, 142(2), 165-189. http://dx.doi.org/10.1111/j.1744-7348.2003.tb00241.x

Fauquet, C., \& Fargette, D. (1990). African cassava mosaic virus: etiology, epidemiology and control. Plant Dis, 74(6), 404-411. http://dx.doi.org/10.1094/PD-74-0404

Food and Agriculture Organization of the United Nations (FAOSTAT). (2009). Please add the title here if available. Retrieved July 26, 2012, from http://faostat.fao.org

Freixenet, J., Muñoz, X., Raba, D., Martí, J., \& Cufí, X. (2002). Yet another survey on image segmentation: Region and boundary information integration. In Computer Vision-ECCV 2002 (pp. 408-422). Springer Berlin Heidelberg. http://dx.doi.org/10.1007/3-540-47977-5_27

Geddes, A. M. W. (1990). The relative importance of crop pests in sub-Saharan Africa. Natural Resources Institute Bulletin, 36, 69. Chatham, UK.

Gonzalez, R. C., \& Woods, R. E. (2010). Digital Image Processing (3rd ed). USA: Pearson.

Hahn, S. K., Terry, E. R., \& Leuschner, K. (1980). Breeding cassava for resistance to cassava mosaic disease. Euphytica, 29(3), 673-683. http://dx.doi.org/10.1007/BF00023215

Haralick, R., \& Shapiro, L. (1985). Image segmentation techniques Computer Vision, Graphics and Image Processing, 29, 32-100. http://dx.doi.org/10.1016/S0734-189X(85)90153-7

Hartigan, J., \& Wang, M. (1979). A K-means clustering algorithm. Applied Statistics, 28, 100-108. http://dx.doi.org/10.2307/2346830

International Institute for Tropical Agriculture (IITA). (1990). Cassava in tropical Africa: A reference manual. Chayce Publication Services, U.K. 
Jain, A., \& Dubes, R. (1988). Algorithms for clustering data. Prentice Hall.

Lloyd, S. (1957). Least squares quantization in PCM's: Bell Telephone Laboratories Paper. Murray Hill.

MacQueen, J. (1967, June). Some methods for classification and analysis of multivariate observations. In Proceedings of the fifth Berkeley symposium on mathematical statistics and probability, 1(14), 281-297.

MATLAB version 7.10.0. (2010). Natick, Massachusetts: The MathWorks Inc., 2010

Nassar, N., \& Ortiz, R. (2010). Breeding cassava to feed the poor. Scientific American, 302(5), 78-84. $\mathrm{http}: / / \mathrm{dx}$. doi.org/10.1038/scientificamerican0510-78

Osiru, D. S. O., Sserubombwe, W. S., Sseruwagi, P., Thresh, M., \& Otim-Nape, G. W. (1999). Effects of Cassava mosaic virus disease on the growth and yield of cassava-some highlights from Makerere experiments. African Crop Science Journal, 7(4), 511-522. http://dx.doi.org/10.4314/acsj.v7i4.27745

Patil, S. B., \& Bodhe, S. K. (2011). Application of Image Processing in Precision Farming. International Journal of Intelligent Information Processing, 4, 137-143.

Sseruwagi, P., Sserubombwe, W. S., Legg, J. P., Ndunguru, J., \& Thresh, J. M. (2004). Methods of surveying the incidence and severity of cassava mosaic disease and whitefly vector populations on cassava in Africa: a review. Virus research, 100(1), 129-142. http://dx.doi.org/10.1016/j.virusres.2003.12.021

Thresh, J. M., \& Cooter, R. J. (2005). Strategies for controlling cassava mosaic virus disease in Africa. Plant pathology, 54(5), 587-614. http://dx.doi.org/10.1111/j.1365-3059.2005.01282.x

Thresh, J. M., Otim-Nape, G. W., Legg, J. P., \& Fargette, D. (1996, August). African cassava mosaic disease: The magnitude of the problem? African J. Root Tuber Crops. Special Issue: Contributions of Biotechnology to Cassava for Africa. In Proceedings of the Cassava Biotechnology Network, Third International Scienti" c Meeting. Kampala, Uganda (Vol. 26, p. 31).

Wang, L., Yang, T., \& Tian, Y. (2008). Crop disease leaf image segmentation method based on color features. In Computer and Computing Technologies In Agriculture, 1, 713-717. Boston, US: Springer. http://dx.doi.org/10.1007/978-0-387-77251-6_78

Wolff, L. B. (1989). Segmentation of Specular Highlights from Object Surfaces. Proc. SPIE 1005, Optics, Illumination, and Image Sensing for Machine Vision III. 198-205. http://dx.doi.org/10.1117/12.949045

\section{Copyrights}

Copyright for this article is retained by the author(s), with first publication rights granted to the journal.

This is an open-access article distributed under the terms and conditions of the Creative Commons Attribution license (http://creativecommons.org/licenses/by/3.0/). 